data heterogeneity and the shortage of applicable studies precludes any firm conclusions being made for clinical practice. Future trials with improved study design (including prospective data collection and consideration of verification bias) may help to further clarify the role of MRI in the assessment and treatment response monitoring of perianal fistulas (particularly in patients with Crohn's disease).

Competing interests None declared.

\section{PTU-119 THE IMPACT OF VARIOUS FACTORS ON BONE LOSS IN IBD PATIENTS TREATED WITH ORAL STEROIDS}

doi:10.1136/gutjnl-2012-302514c.119

M Y Gherghab, ${ }^{*}$ P Sahay. Gastroenterology, Mid Yorkshire Hospitals, Wakefield, UK

Introduction Many factors can contribute to bone loss in Inflammatory bowel disease (IBD) patients treated with oral steroids.

Methods To assess the prevalence and evaluate the impact of various factors on bone density in IBD patients treated with oral steroids. We conducted a retrospective study on 50 patients with ulcerative colitis (UC) and 40 patients with Crohn's disease. BMD of lumbar spine and femoral neck were measured by axial dual-energy x-ray absorpiometry scan (DEXA) in 57 patients, and that of forearm by peripheral DEXA scan in 33 patients.

Results $60 \%$ of all patients $(n=55)$ had low BMD (8.8\% were osteoporotic, $51 \%$ were osteopenic). The osteoporotics were predominantly $(75 \%)$ patients with $\mathrm{CD}$ and were smokers. On the other hand, $63 \%$ of osteopenics had UC and $28 \%$ were smokers. Although most of males (80.7\%) had low BMD (73.3\% were aged $<50$ years), only one third of females below the age of 45 years had low BMD. Only five patients had BMI $<19$, the majority of these patients $(80 \%)$ had low BMD. All of the osteoporotic CD patients had terminal ileum (TI) involvement, and nearly $60 \%$ of patients who underwent surgery for $\mathrm{CD}$ had low bone mineral density. Patients who had IBD for 10 years showed low BMD in $62.5 \%$, while $54 \%$ of those with disease duration more than 10 years had low BMD. $40 \%$ of patients who were on steroid sparing agents had normal BMD. All of the osteoporotic female patients were aged $>45$ years and were not on bone protection.

Conclusion The high prevalence of bone loss in IBD patients treated with oral steroids is multifactorial. Disease type (CD), site of the disease (TI), disease severity (requiring oral steroids and surgical intervention) and low BMI seems to be the major variables and early bone protection is recommended especially in young men.

Competing interests None declared.

\section{PTU-120 A REVIEW OF PATIENTS IN A SINGLE CENTRE WITH ILEAL POUCH-ANAL ANASTOMOSIS FOR ULCERATIVE COLITIS AND AN ASSESSMENT OF THOSE PATIENTS WHO REQUIRE ON GOING MEDICAL THERAPY}

doi:10.1136/gutjnl-2012-302514c.120

${ }^{1} \mathrm{~N}$ Allen, ${ }^{*}{ }^{2} \mathrm{E}$ Garforth, ${ }^{2} \mathrm{R}$ Heath, ${ }^{1} \mathrm{P}$ Rooney. ${ }^{1}$ General Surgery, The Royal Liverpool and Broadgreen Teaching Hospital, Liverpool, UK; ${ }^{2}$ Surgery, Royal Liverpool and Broadgreen Teaching Hospital, Liverpool, UK

Introduction Restorative proctocolectomy with ileal pouch-anal anastomosis (IPAA) is the surgical therapy of choice for patients with chronic ulcerative colitis. However IPPA is frequently accompanied by early and late complications. A proportion of patients require ongoing medical management which this study assesses.

Methods A prospectively collected hospital database of patients who were followed up after IPAA at a single centre was retrospectively reviewed. A review of all case notes was carried out to assess surgical intervention prior to IPAA. All post operative complications were recorded and an assessment was made of those patients recommenced on medical therapy.

Results 102 patients' case notes were reviewed, (60 male: 42 female mean age 42 years SD of \pm 12.01$)$. The follow-up is ongoing and is currently between 2 and 193 months. The complications included anastomotic leak $(n=4)$, incisional hernia $(n=7)$, pouchitis $(n=36)$, stenosis $(n=15)$, pre pouch stricture $(n=1)$, ileitis $(n=2)$, enterocutaneous fistula $(n=2)$, perianal fistula $(n=6)$, pouch vaginal fistula $(n=5)$, pouch ulceration $(n=8)$. All patients prior to surgery had a histological diagnosis of ulcerative colitis, 4 patients were reclassified as having Crohn's or indeterminate colitis at further follow-up. There were $13(12.7 \%)$ patients whom were recommenced on medical therapy, including sulphasalazine, budesonide, azathioprine, 6-mercaptopurine and infliximab. Patients who were on antibiotics were not included in these numbers. All patients who were reclassified as having Crohn's were recommenced on medical therapy and seen in a joint gastro/surgical clinic.

Conclusion Long term anastomotic problems are common after IPPA. Most pouch patients do not require additional medical treatment other than antibiotics but $13 \%$ need continued complex medical therapy under the care of gastroenterologists and surgeons. Use of steroids is low. Diagnostic problems remain an issue.

Competing interests None declared.

\section{PTU-121 COMPLIANCE WITH GUIDELINES ON VIRAL SCREENING AND VACCINATION OF PATIENTS WITH INFLAMMATORY BOWEL DISEASE (IBD)}

doi:10.1136/gutjnl-2012-302514c.121

N Halliday, ${ }^{*}$ S Murray, G Parkes, V S Wong. Department of Gastroenterology, Whittington Hospital, London, UK

Introduction ECCO recommends screening IBD patients for immunity to or infection with varicella zoster virus (VZV), hepatitis $\mathrm{B}(\mathrm{HBV})$ and potentially HIV, hepatitis $\mathrm{C}$ virus (HCV) and tuberculosis (TB) to allow monitoring or treatment if patients require immunomodulatory therapy. Patients should be offered vaccination against VZV, HBV, human papilloma virus (HPV), pneumococcus and influenza where appropriate. We audited screening practice and the reported prevalence of prior exposure and vaccination in our IBD population.

Methods In 2010, IBD patients in our general gastroenterologyclinics completed a questionnaire regarding prior VZV disease, HBV infection or vaccination, influenza, pneumococcal and HPV vaccination. Results for screening tests were checked.

Results Patient characteristics: 91 patients returned questionnaires. 46 were male; median age was 43 years (range 19-71). 61 had a diagnosis of ulcerative colitis, 30 of Crohn's disease. Current drug therapy included none in 13 patients, 5-aminosalicylic acid (5-ASA) drugs in 42 , systemic corticosteroids in 6 , purine analogues, antimetabolites or calcineurin inhibitors in 25 and biological agents in 5 . Questionnaire responses: see Abstract PTU-121 table 1. Screening: 70 patients were screened for VZV immunity and 10 had complete HBV screening (surface antibody, antigen and anti-core antibody). Levels of screening for HCV, HIV were low (18 and 3 patients respectively). TB screening was more comprehensive; 47 had chest $\mathrm{x}$-rays and 2 had $\gamma$ interferon release assays. Those on biological agents were more likely to have been screened for TB. Four patients reporting prior VZV disease had no evidence of immunity (out of 48 who were tested). Nine of the 15 reporting HBV vaccination were tested, 5 of whom were negative. The one patient with serology consistent with previous HBV infection had no recollection of such. Conclusion Overall compliance with ECCO guidelines was low and sporadic among our population. Other groups have reported similar 
low rates. Low levels of patients are taking advantage of existing immunisation programmes. Patient recall of previous vaccination may not be sufficient to imply immunity. A systematic process for assessing patients' vaccination status and active encouragement of vaccination programmes should be undertaken in the outpatient setting.

Abstract PTU-121 Table 1 Questionnaire responses

\begin{tabular}{|c|c|c|c|c|c|}
\hline$n=91$ & $\begin{array}{l}\text { VZV } \\
\text { disease }\end{array}$ & $\begin{array}{l}\text { HBV infection } \\
\text { or vaccination }\end{array}$ & $\begin{array}{l}\text { Pneumococcal } \\
\text { vaccine }\end{array}$ & $\begin{array}{l}\text { Influenza } \\
\text { vaccine }\end{array}$ & $\begin{array}{l}\text { HPV vaccine } \\
(n=45)\end{array}$ \\
\hline Yes & $63(69 \%)$ & $15(16 \%)$ & $8(9 \%)$ & $28(31 \%)$ & $0(0 \%)$ \\
\hline No & $10(11 \%)$ & $58(64 \%)$ & $78(86 \%)$ & $61(67 \%)$ & $37(82 \%)$ \\
\hline Unknown & $18(20 \%)$ & 17 (19\%) & $4(4 \%)$ & $2(2 \%)$ & 7 (16\%) \\
\hline No answer & - & $1(1 \%)$ & $1(1 \%)$ & - & $1(2 \%)$ \\
\hline $\begin{array}{l}\text { Complete } \\
\text { screening }\end{array}$ & $70(77 \%)$ & $10(11 \%)$ & NA & NA & NA \\
\hline
\end{tabular}

Competing interests None declared.

\section{PTU-122 A TRIAL WITH MERCAPTOPURINE FOLLOWING AZATHIOPRINE INTOLERANCE IS A SAFE TREATMENT STRATEGY FOR THE MAJORITY OF PATIENTS WITH IBD}

doi:10.1136/gutjnl-2012-302514c.122

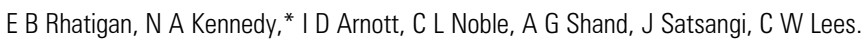
Gastrointestinal Unit, Molecular Medicine Centre, Western General Hospital, Edinburgh, UK

Introduction Azathioprine intolerance (AZA-I) leads to withdrawal of therapy in up to $30 \%$ of patients with IBD. Smaller case series demonstrated that mercaptopurine (MP) could be a well tolerated alternative in selected patients. ${ }^{1}$ This study aims to further assess its tolerability, in a larger cohort of AZA-I patients, over a longer period of time, and to re-evaluate potential factors predictive of tolerance. Methods A retrospective audit was made of 137 patients with IBD (78 women, median age at diagnosis 32 years, 78 with CD, 59 with UC) who had been intolerant of AZA and then subsequently treated with MP

Results MP was tolerated by $58 \%$ of AZA-I patients (median followup 937 days, median dose $0.91 \mathrm{mg} / \mathrm{kg}$ ). Tolerance was highest in patients with AZA related gastrointestinal intolerance $(66 \%)$ and hepatotoxicity (61\%), and lowest in patients with AZA related flulike illness (40\%). The number of patients with AZA induced neutropenia and pancreatitis were too small to draw firm conclusions (see Abstract PTU-122 table 1). Age at diagnosis was significantly associated with tolerability. Patients intolerant of MP were younger ( 28 vs 33 yro; $p=0.024$ ) and of those under the age of 40 only $55 \%$ tolerated MP compared with $69 \%$ of those aged 40 years or over ( $\mathrm{p}=$ Azathioprine-intolerant patients, subsequently treated with mercaptopurine (AZA-I/MP) grouped by azathioprine intolerance

Conclusion Consistent with previous data, this, the largest series to date, with substantial follow- up, has shown that MP is a safe alternative for up to $60 \%$ of AZA-I patients, including some with a previous major intolerance. Patients with previous gastrointestinal intolerance or hepatotoxicity may be more likely to tolerate a trial of MP.

Competing interests None declared.

\section{REFERENCE}

1. Lees, et al. Aliment Pharmacol \& Ther 2007;27:220-7.

\section{PTU-123 ACUTE SEVERE ULCERATIVE COLITIS: THE LAST 12 YEARS IN EDINBURGH}

doi:10.1136/gutjnl-2012-302514c.123

N A Kennedy, ${ }^{*}$ J E Van Ross, N C Hare, G-T Ho, H E Drummond, A G Shand, I D Arnott, C W Lees, J Satsangi. Gastrointestinal Unit, Molecular Medicine Centre, Western General Hospital, Edinburgh, UK

Introduction Acute severe ulcerative colitis (ASUC) remains a common reason for urgent colectomy, yet there are relatively few large cohort studies exploring prognosis and outcome. ${ }^{1-3}$ This study aims to examine presentation and management of ASUC in the Western General Hospital, a tertiary referral centre in Edinburgh and to identify prognostic factors.

Methods Patients were identified from a large database of participants in genetic studies in Edinburgh, as well as from two previous small cohorts of ASUC studied in Edinburgh. More recent cases were found from minutes of a weekly IBD meeting. Cases were included if they met the standard clinical, radiological and pathological criteria for ulcerative colitis and required an admission for 3 days or more requiring intravenous corticosteroids and/or colectomy. Two cohorts were analysed, one with full clinical detail (97 admissions in 86 patients) and one with more basic detail (444 admissions in 323 patients).

Results Overall colectomy rate was $31.8 \%$. Haemoglobin, $\mathrm{C}$ reactive protein and albumin at days 0 and 3 were significant predictors of colectomy in both cohorts ( $p<0.05$ in each case), while in the detailed cohort day 3 but not day 0 stool frequency was predictive ( $p<0.001$ and 0.81 respectively). A simple score was derived to predict colectomy at admission based on disease extent, albumin and CRP. Scores of 0, 1, 2 and 3 corresponded to risks for colectomy

Abstract PTU-122 Table 1

\begin{tabular}{|c|c|c|c|c|c|c|c|c|c|}
\hline \multirow[b]{2}{*}{$\begin{array}{l}\text { Type of azathioprine } \\
\text { intolerance }\end{array}$} & \multirow[b]{2}{*}{$\mathbf{n}$} & \multirow{2}{*}{$\begin{array}{l}\text { Proportion with } \\
\text { mercaptopurine } \\
\text { tolerance (\%) }\end{array}$} & \multicolumn{6}{|c|}{ Type of mercaptopurine intolerance } & \multirow{2}{*}{$\begin{array}{l}\text { Proportion of patients where } \\
\text { intolerance of mercaptopurine } \\
\text { same as for azathioprine (\%) } \\
30 / 55(55 \%)\end{array}$} \\
\hline & & & $\begin{array}{l}\text { GI } \\
\text { Intolerance }\end{array}$ & $\begin{array}{l}\text { Flu-like } \\
\text { illness }\end{array}$ & Hepatotoxicity & Neutropenia & Pancreatitis & Other & \\
\hline GI Intolerance* & 67 & $44 / 67(66 \%)$ & 14 & 2 & 0 & 5 & 2 & 0 & $14 / 23(61 \%)$ \\
\hline Flu-like illness & 35 & $14 / 35(40 \%)$ & 4 & 10 & 3 & 2 & 0 & 2 & $10 / 21(48 \%)$ \\
\hline Hepatotoxicity & 18 & $11 / 18(61 \%)$ & 1 & 1 & 2 & 2 & 0 & 1 & $2 / 7(29 \%)$ \\
\hline Neutropenia & 4 & $3 / 4(75 \%)$ & 0 & 0 & 0 & 1 & 0 & 0 & $1 / 1(100 \%)$ \\
\hline Pancreatitis & 3 & $2 / 3(67 \%)$ & 0 & 0 & 0 & 0 & 1 & 0 & $1 / 1(100 \%)$ \\
\hline Other $†$ & 8 & $6 / 8(75 \%)$ & 0 & 0 & 0 & 0 & 0 & 2 & $2 / 2(100 \%)$ \\
\hline Unknown & 2 & $0 / 2(0 \%)$ & 1 & 1 & 0 & 0 & 0 & 0 & NA \\
\hline
\end{tabular}

*Nausea, vomiting, abdominal pain, diarrhoea.

†Rash, Alopecia, Headaches, Itch, anaemia and acute kidney injury, acne. 